Check for updates

Cite this: Mater. Adv., 2020 1,1858

Received 5th June 2020

DOI: $10.1039 / \mathrm{d} 0 \mathrm{ma} 00382 \mathrm{~d}$

rsc.li/materials-advances Accepted 16th July 2020

\section{Asymmetric-donor $\left(D_{2} D_{2}{ }^{\prime}\right)$-acceptor $(A)$ conjugates for simultaneously accessing intrinsic blue-RTP and blue-TADF $\dagger$}

\begin{abstract}
Harsh Bhatia and Debdas Ray (D) *
The development of new photoluminescent (PL) materials with simultaneous room-temperature phosphorescence (RTP) and thermally activated delayed fluorescence (TADF) features is highly desirable for bio-imaging, security applications and sensors due to the involvement of both singlet and longerlived triplet states. Here, we report two carbazolyl-phenoxy-phthalonitrile conjugates (CPPN, CPPNF). Spectroscopic studies combining two daughter compounds (PPN, PPNF) in polar and nonpolar hosts confirmed efficient blue-RTP from the higher-energy triplet state ( $\left.T_{\text {PPN }}\right)$ due to the phenoxyphthalonitrile (PPN) part, and blue-TADF via reverse intersystem crossing from the low-lying triplet state $\left(T_{C z P N}\right)$ of the carbazolyl-phthalonitrile $(\mathrm{CzPN})$ part to the singlet $\left(\mathrm{S}_{1}\right)$ state of the same CzPN part, utilizing the $\mathrm{T}_{\mathrm{PPN}}$ state that acts as an intermediate for spin-vibronic coupling. Such PL characteristics are observed due to the energetic proximity of ${ }^{3} \mathrm{LE}_{\mathrm{PPN}},{ }^{1} \mathrm{CT}_{\mathrm{CZPN}}$ and ${ }^{3} \mathrm{CT}_{\mathrm{CZPN}}$. In the hydrogen-bonded matrix and crystals, we found faint persistent green-RTP characteristics of the PPNF due to supramolecular interactions and aggregation of the molecule. This study could pave the way to understanding the involvement of different excited states associated with TADF and RTP processes of asymmetric-donoracceptor systems.
\end{abstract}

\section{Introduction}

Single-component organic luminescent materials (SCOLMs) that show emission features with high photoluminescence quantum yield (PLQY) utilizing both singlet and triplet excited states have attracted increasing attention for their potential use in organic chemical sensing, ${ }^{1,2}$ and bio-imaging ${ }^{3}$ (Fig. 1). Efficient emission via fluorescence ${ }^{4}$ can occur from the singlet excited states where the relaxation conserves spin. On the other hand, phosphorescence from the lowest triplet $\left(\mathrm{T}_{1}\right)$ state is typically very weak because it involves a spin flip, which is quantum mechanically forbidden. ${ }^{5}$ Therefore, a search for emitters that show simultaneous radiative decay of both singlet and triplet states and high PLQY are of particular interest.

Recently, organic donor-acceptor (D-A) based emitters with a thermally activated delayed fluorescence (TADF) ${ }^{6-11}$ feature that occurs via reverse intersystem crossing (rISC) from either the higher lying triplet state $\left(\mathrm{T}_{n}, n>1\right)^{6-8}$ or the lowest triplet $\left(\mathrm{T}_{1}\right)$ state

Advanced Photofunctional Materials Laboratory, Department of Chemistry, Shiv Nadar University, NH-91, Tehsil Dadri, District Gautam Buddha Nagar, Uttar Pradesh, 201 314, India.E-mail: debdas.ray@snu.edu.in

$\dagger$ Electronic supplementary information (ESI) available: Synthesis, NMR spectra, additional photophysical analysis and single-crystal X-ray data. CCDC 1990136 and 1990137. For ESI and crystallographic data in CIF or other electronic format see DOI: 10.1039/d0ma00382d

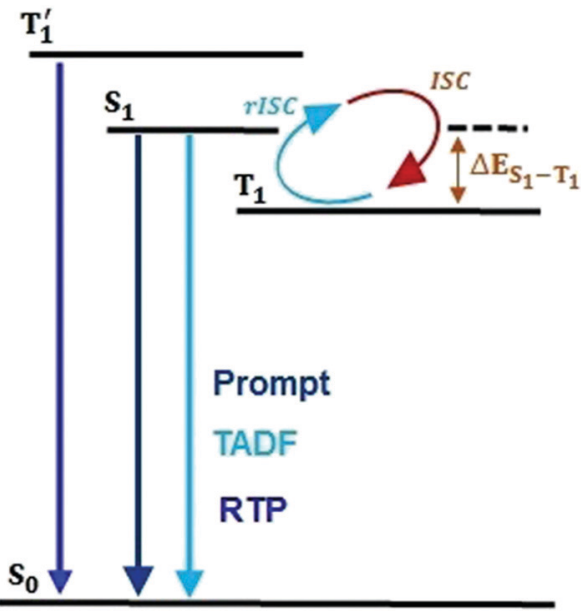

Fig. 1 Jablonski diagram for emission via RTP and TADF in the proposed molecular design.

to the lowest singlet $\left(\mathrm{S}_{1}\right)$ state, ${ }^{9-11}$ have showed exciting capabilities for energy-efficient light emission by harvesting of both singlet and triplet states. To harness the triplet exciton via TADF, numerous research studies have been undertaken, which have established that apart from the low $\mathrm{S}_{1}-\mathrm{T}_{1}$ gap between the singlet and triplet states, the nonadiabatic effect between the low-lying excited states 
along with mixing of the intermediate excited states plays a crucial role in the process of ISC and rISC. ${ }^{12-17}$ On the other hand, the efficient room-temperature phosphorescence (RTP) from the triplet state of heavy-metal based systems has been widely investigated. ${ }^{18-22}$ These systems show up to $100 \%$ internal electroluminescence efficiency due to the use of noble metals allowing strong spin-orbit coupling (SOC). ${ }^{18}$ Despite their high efficiency electroluminescence, these systems are both expensive and toxic. An alternative approach to substitute such systems with cheaper organic materials which can be easily processed and functionalized using a wide variety of substituents, is therefore desirable. Recently, purely organic RTP materials ${ }^{23-29}$ have boosted tremendous interest in data security protection ${ }^{30}$ and invisible ink, ${ }^{31}$ and photodynamic therapy. ${ }^{29}$ However, these systems show weak RTP due to inefficient ISC between the $S_{1}$ and $T_{1}$ states caused by the low SOC of the lighter elements present in the dye. ${ }^{32-35}$ In order to enhance the ISC rate, heteroatoms (N, O, S) and halogens are introduced as they can provide radiationless ISC transition between the orbitals of different symmetries i.e. ${ }^{1}\left(\pi-\pi^{*}\right)$ to ${ }^{3}\left(\mathrm{n}-\pi^{*}\right)$ and vice versa (El-Syed rule). ${ }^{34}$ However, the RTP efficiency of such molecular systems is very low due to the slow rate of ISC that causes a high lifetime of the triplet states. In addition, the molecular vibrations that readily increase the non-radiative pathways of the localized triplet excitons become another major bottleneck to harvest efficient phosphorescence yield. ${ }^{36}$ To circumvent the above issues, crystal formation, ${ }^{37,38}$ aggregation, ${ }^{39,40}$ hostguest interactions ${ }^{41}$ and deuteration ${ }^{42}$ have been studied to reduce the nonradiative pathways under ambient conditions. To observe both blue-TADF (BTADF) and blue-RTP (BRTP) simultaneously with high PLQY from the SCOLMs under ambient conditions becomes a difficult task in the field of chemical physics. To harvest triplet excitons simultaneously from a single molecule, fine-tuning of the singlet-triplet energy gap is required for both TADF and RTP, while intra- and/or intermolecular interactions (lp $\cdots \pi, \pi \cdot \pi$, hydrogen bonding $)^{27,30}$ and heavy elements are the prerequisites for RTP. ${ }^{19}$ Recently, several design principles have been used to observe both TADF and RTP. Huang et al. synthesized angular and linear isomers, and tuned the energy gaps using the host materials to harvest triplet excitons through TADF and RTP. ${ }^{43}$ Ward et al. showed TADF and RTP separately through chemical modification. ${ }^{44}$ Additionally, mechanical force, ${ }^{45,46}$ structural modification, ${ }^{47}$ conformational switching ${ }^{48}$ and change in host matrices ${ }^{49}$ have successfully been used to demonstrate both TADF and RTP. Despite these achievements, the major issue is the lack of a proper structural design and understanding of the origin of simultaneous BRTP and BTADF in a single molecule under ambient conditions. In addition, to observe such emission features with persistent-RTP (PRTP), the materials need to show longerlived triplet emissions under ambient conditions in host matrices. Due to these bottlenecks, the mechanism of simultaneously observing BRTP and BTADF along with PRTP in the SCOLMs under ambient conditions remains murky. Hence, the development of such SCOLMs is of prime importance. Taking a cue from our previous reports of $\mathrm{D}_{4}-\mathrm{A}$ molecular systems ${ }^{30,31}$ where the phenoxy or substituted phenoxy donors are covalently attached to either the phthalonitrile (PN) or the terephthalonitrile ring, here

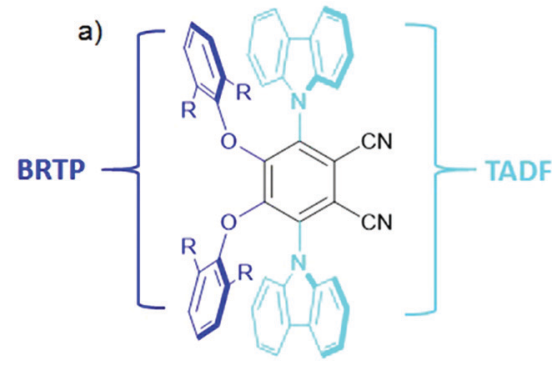

b)
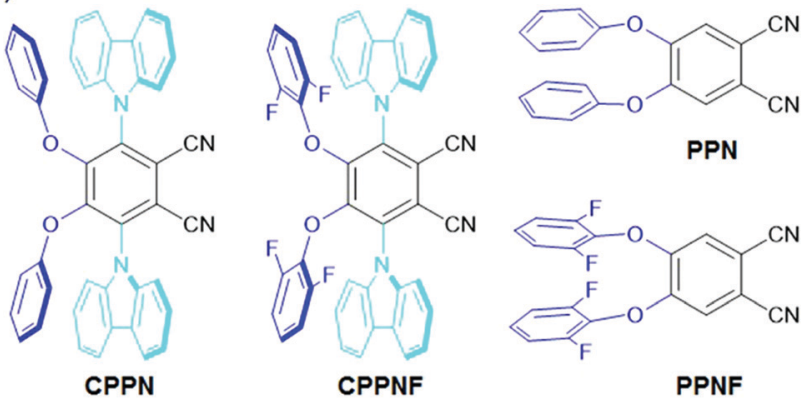

Fig. 2 (a) Design strategy for creating materials with simultaneous blueRTP and blue-TADF. (b) Structure of the molecules (CPPN and CPPNF) including the two control compounds (PPN and PPNF) synthesized in this study.

we attached both phenoxy or 2,5-difluorophenoxy and carbazole donors at the 4,5 and 3,6-positions of the PN core respectively, to get mixed donor-acceptor $\left(\mathrm{D}_{2} \mathrm{D}_{2}{ }^{\prime}-\mathrm{A}\right)$ geometries (CPPN, CPPNF) (Fig. 2). We hypothesized that incorporation of asymmetric donors in the PN core would play a crucial role in observing both BRTP and BTADF due to the involvement of the $\mathrm{T}_{\mathrm{PPN}}$ state caused by the phenoxy-phthalonitrile (PPN) part and upconversion of the triplet state $\left(\mathrm{T}_{\mathrm{CZPN}}\right)$ to the singlet state $\left(\mathrm{S}_{\mathrm{CzPN}}\right)$ caused by the traditional carbazolyl-phthalonitrile (CzPN) part. The possible advantage of such a system with asymmetric donors motivated us to access the role of the intermediate state in the RTP and TADF processes. Furthermore, 2,5-difluorophenoxy donors are selected because they may favor both SOC via $\operatorname{lp}(\mathrm{O} / \mathrm{F}) \cdots \pi$ interactions due to the presence of multiple $\mathrm{O}$ and $\mathrm{F}$ atoms in the molecular backbone and hydrogen-bonding (H-B) interactions with the host to facilitate faint persistent-RTP. Our studies revealed that both compounds (0.1\% poly(methyl methacrylate), PMMA) under ambient conditions show both-BRTP (CPPN: phosphorescence quantum yield, $\Phi_{\mathrm{P}}=3 \%$; CPPNF: $\Phi_{\mathrm{P}}=3.5 \%$ ) and BTADF (CPPN: fluorescence quantum yield, $\Phi_{\mathrm{F}}=43 \%$; CPPNF: $\Phi_{\mathrm{F}}=44 \%$ ) due to the radiative decay of the upper triplet $\left(\mathrm{T}_{\mathrm{PPN}}\right)$ state (higher-energy) and rISC from the lower-lying triplet $\left(\mathrm{T}_{\mathrm{CzPN}}\right)$ state to the singlet $\left(\mathrm{S}_{\mathrm{CzPN}}\right)$ state. In contrast to PMMA, bis[2-(di-(phenyl)phosphino)-phenyl]ether oxide (DPEPO) films (0.1\%) of both compounds showed an increase in $\Phi_{\mathrm{P}}(\sim 10 \%)$ while a decrease in $\Phi_{\mathrm{F}}(\sim 10 \%)$ was observed. Moreover, photoluminescence (PL) studies of CPPNF in both polyvinyl alcohol (PVA) matrix and crystals revealed faintgreen-persistent RTP characteristics under ambient conditions.

Considering two control compounds we speculate that the BRTP originates due to the PPN part of the molecule while BTADF occurs due to the vibronic coupling between the lowest 
$\mathrm{S}_{1}(\mathrm{CT})(\mathrm{CzPN})$ and $\mathrm{T}_{1}(\mathrm{CT})(\mathrm{CzPN})$ states via the upper $\mathrm{T}_{\mathrm{PPN}}\left({ }^{3} \mathrm{LE}\right)$ state. The photoluminescence studies of CPPNF in the hydrogen-bond (H-B) matrix further show that it exhibits PRTP with lifetimes of $39.51 \mathrm{~ms}$ and $269.21 \mathrm{~ms}$.

\section{Experimental}

\subsection{Synthesis and characterization}

The compounds were synthesized via the traditional nucleophilic aromatic substitution reaction $\left(\mathrm{S}_{\mathrm{N}} \mathrm{R}\right)$ between the phenol derivatives and 3,4,5,6-tetrafluorophthalonitrile. ${ }^{30,31}$ The isolated intermediates were reacted separately with the sodium salt of carbazole to obtain the final compounds (CPPN, CPPNF) (Fig. 2). Control compounds (PPN, PPNF) were synthesized following a similar method. All the compounds were characterized using NMR spectroscopy, HRMS, and X-ray analysis (see the ESI $\dagger$ ).

\subsection{Photophysical measurements}

Absorption measurements were performed using a Cary 8454 UV-vis instrument from Agilent Technologies. Steady-state emission, phosphorescence, and time resolved analysis of the samples were recorded on Horiba Fluorolog. All the phosphorescence spectra were recorded by giving the detector a delay of $0.05 \mathrm{~ms}$. The measurement details can be found in the reported literature. ${ }^{8,32,33}$ Temperature-dependent PL measurements were performed using a liquid $\mathrm{N}_{2}$ Dewar assembly. All the energy levels have been calculated from the onset of the emission spectra.

\section{Results and discussion}

\subsection{X-ray crystallography}

X-ray analysis (Fig. 3) of both compounds revealed that two adjacent phenoxy donors are covalently attached to the 4,5-positions of the PN core with torsions of $-54.51^{\circ}$ (CPPN) and $-50.39^{\circ}$ (CPPNF) when viewed along the $\mathrm{C}(2)-\mathrm{C}(3)-\mathrm{O}(1)-$ $\mathrm{C}(17)$ atoms while torsions of $120.83^{\circ}$ (CPPN) and $120.48^{\circ}$ (CPPNF) viewed along the atoms $\mathrm{C}(1)-\mathrm{C}(2)-\mathrm{N}(2)-\mathrm{C}(5)$ are measured when the 3,6-positions of the PN are substituted with the carbazolyl rings. Interestingly, the two phenyl rings in both CPPN and CPPNF are in face-to-face orientation with the carbazolyl donors (centroid-to-centroid; CPPN, $3.608 \AA$ ^ CPPNF, 3.600 ̊̊). Furthermore, strong intramolecular $\operatorname{lp}(\mathrm{F}) \cdots \pi$ (centroid) interactions (3.270 $\mathrm{A}$ ) are also observed. Thus, X-ray analysis unambiguously confirms our structural model.

\subsection{Absorption and emission characteristics in solutions}

Fig. S5 (ESI $\dagger$ ) shows the normalized ultraviolet-visible spectra of both CPPN and CPPNF in the solvents of disparate polarity $(10 \mu \mathrm{M})$. Both compounds exhibit higher energy absorption bands at $285 \mathrm{~nm}, 315 \mathrm{~nm}$ and $325 \mathrm{~nm}$ which can be attributed to the $\pi-\pi^{*}$ transition. In addition, a broad tail (340-425 nm), which can be assigned to charge transfer (CT), is accompanied by the former bands. Likewise, steady-state emissions of both compounds were recorded in the solvents of disparate polarity $\left(\lambda_{\mathrm{ex}}=350 \mathrm{~nm}, 10 \mu \mathrm{M}\right)$ (Fig. 4 and Fig. S6, ESI $\dagger$ ). Emission spectra
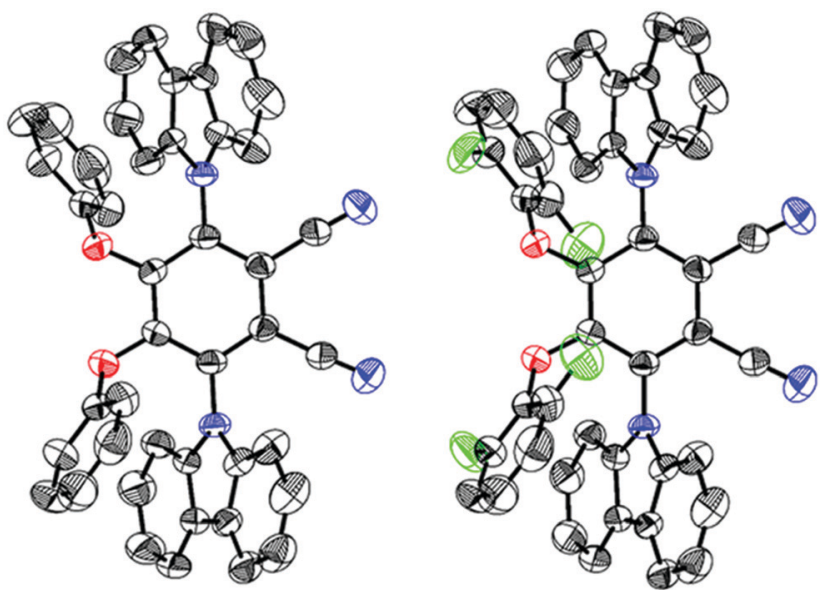

Fig. 3 Oak Ridge thermal ellipsoid plots (50\% probability ellipsoids) of CPPN (left) and CPPNF (right). The protons are removed for the sake of clarity.

of both compounds show a gradual batho-chromic shift of the emission maxima along with reduced intensity of the broad emission band with increasing solvent polarity. Such emission features confirm that the excited state is CT in nature. Furthermore, substantial enhancement of emission intensity as well as lifetimes recorded in deoxygenated toluene solutions suggest an involvement of the triplet state that relaxes radiatively from the same singlet excited state (Fig. 4 and Fig. S7, ESI $\dagger$ ). Moreover, the biexponential decay of both compounds (CPPN: $\lambda_{\mathrm{em}}=473 \mathrm{~nm}$, $\tau_{\mathrm{PF}}=9.65 \mathrm{~ns}, \tau_{\mathrm{DF}}=331.92 \mathrm{~ns} ;$ CPPNF: $\lambda_{\mathrm{em}}=477 \mathrm{~nm}, \tau_{\mathrm{PF}}=17.26 \mathrm{~ns}$, $\left.\tau_{\mathrm{DF}}=1.63 \mu \mathrm{s}\right)$ under ambient conditions unambiguously confirms the TADF feature.

\subsection{Emission characteristics in PMMA films}

To elaborate our understanding of the emission characteristics in rigid medium, photophysical studies were performed in the
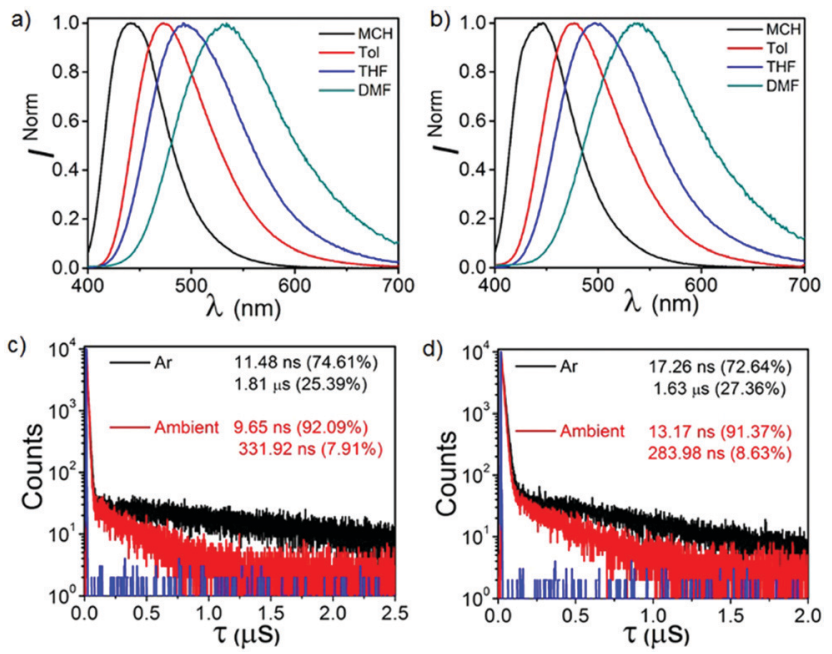

Fig. 4 Solvatochromic emissions of (a) CPPN and (b) CPPNF under ambient conditions. Lifetime analysis of (c) CPPN and (d) CPPNF in toluene under ambient and deoxygenated conditions. 
poly(methyl methacrylate) (PMMA) matrix $(0.1 \% \quad(\mathrm{w} / \mathrm{w}))$ $\left(\lambda_{\mathrm{ex}}=350 \mathrm{~nm}\right)$. It should be noted that the energy of all the emission peaks were calculated taking the on-set of the spectra. Both CPPN and CPPNF show broad emission bands at $463 \mathrm{~nm}$ in PMMA films with photoluminescence quantum yields (PLQY) of $47.5 \%$ and $45.3 \%$, respectively (Fig. 5). We found that emission intensity of the $\lambda_{463}$ significantly increases under argon environment at RT, indicating a triplet state involvement for the emission (Fig. S8, ESI $\dagger$ ). The emission peak at $463 \mathrm{~nm}$ for both cases is assigned as typical TADF, which was confirmed by the temperature-dependent lifetime measurements (Fig. 5, Fig. S9 and Table S1, ESI $\dagger$ ). Furthermore, time resolved emission spectra (TRES) of both compounds were collected from $3.2 \mathrm{~ns}$ to $49.3 \mathrm{~ns}$ at RT. The emission spectra continuously red shifts from $450 \mathrm{~nm}$ (onset, $3.10 \mathrm{eV}$ ) to $465 \mathrm{~nm}$ (onset, $3.03 \mathrm{eV}$ ) in both cases (Fig. 6) due to the relaxation of the excited states, while no further red shift was observed in $34 \mathrm{~ns}$ onward, which indicates that no other lower energy emissive states are present at RT (Fig. S10, ESI $\dagger$ ). The phosphorescence measurements were performed with a high detector delay (0.05 ms) to remove the DF component. Surprisingly, phosphorescence measurements of both compounds under ambient conditions exhibit blue shifted narrow RTP bands at $455 \mathrm{~nm}$ (CPPN: $3.06 \mathrm{eV}, \tau=58.49 \mu \mathrm{s}, 175.06 \mu \mathrm{s}, 619.3 \mu \mathrm{s}, \Phi_{\mathrm{P}}=3.26 \%$ ) and $452 \mathrm{~nm}$ (CPPNF: $3.05 \mathrm{eV}, \tau=148.66 \mu \mathrm{s}, 570.09 \mu \mathrm{s}, 44.58 \mu \mathrm{s}$, $\Phi_{\mathrm{P}}=2.24 \%$ ) with a FWHM of $\sim 67 \mathrm{~nm}$ (Fig. 5 and Table 1) as compared to the FWHM of $80 \mathrm{~nm}$ (CPPN) and $78 \mathrm{~nm}$ (CPPNF) for delayed fluorescence, indicating different natures of excited states. Moreover, temperature-dependent lifetime measurements show a gradual enhancement of phosphorescence lifetimes $^{50}\left(\tau_{\mathrm{p}}\right)$ with lowering of temperature, indicating BRTP characteristics of the molecules (Table S2 and Fig. S9, ESI $\dagger$ ). Interestingly, at $77 \mathrm{~K}\left(\lambda_{\text {ex }}=350 \mathrm{~nm}\right)$, both steady state and phosphorescence emission bands of the compounds closely
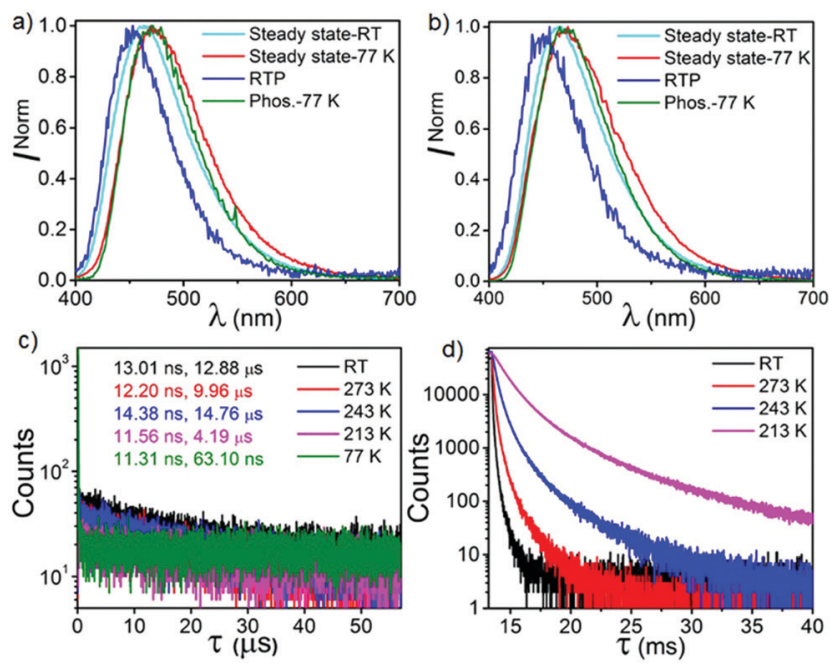

Fig. 5 Steady-state and phosphorescence spectra of (a) CPPN and (b) CPPNF at RT (ambient conditions) and $77 \mathrm{~K}$ in PMMA (0.1\%). Temperature-dependent (c) fluorescence and (d) phosphorescence decay analysis of CPPN in $0.1 \%$ PMMA.
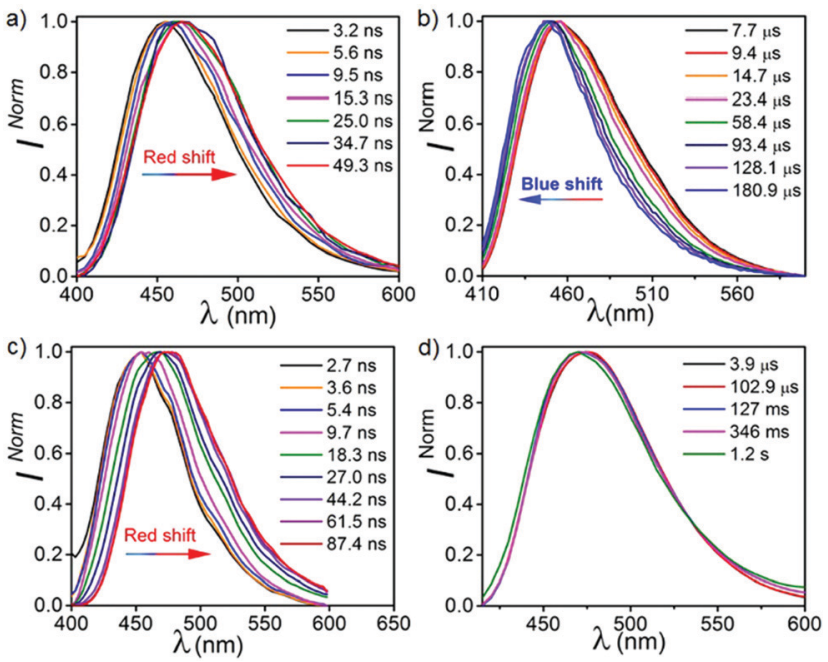

Fig. 6 TRES of CPPN (a) without detector delay and (b) $6.8 \mu$ s detector delay under ambient conditions. TRES of CPPN (c) without detector delay and (b) varying detector delay from 0.034 to $110 \mathrm{~ms}$ at $77 \mathrm{~K}$.

overlap ( $\sim 473 \mathrm{~nm}$, red shifted), and they appear as a broadband with an on-set of 2.95 (CPPN) and $2.98 \mathrm{eV}$ (CPPNF), indicating an involvement of the ${ }^{3} \mathrm{CT}$ state (Fig. 5). The disappearance of the high-energy band $(\sim 455 \mathrm{~nm})$ at $77 \mathrm{~K}$ is discussed in a later section of the manuscript. Comparing emission and decay analysis recorded at both RT and $77 \mathrm{~K}$, we confirm that two triplet states contribute to the emission under ambient conditions.

To understand the number of excited states involved in the process, TRES measurements were also performed at $77 \mathrm{~K}$.

These studies show that the emission peaks of both compounds bathochromically shift from 455 to $475 \mathrm{~nm}$ (Fig. 6 and Fig. S11, ESI $\dagger$ ). When we compare these results with the TRES data recorded at RT, we found that the spectrum obtained at $\sim 2 \mathrm{~ns}$ is closely overlapped by the unrelaxed TRES data at RT (3.2 ns) (Fig. S11, ESI $\dagger$ ). Interestingly, on a later time scale $(\sim 45 \mathrm{~ns})$ at $77 \mathrm{~K}$, a relatively larger bathochromic shift of the emission spectra was recorded $(475 \mathrm{~nm}, 2.98 \mathrm{eV})$ as compared to the spectra recorded at RT ( $465 \mathrm{~nm}, 3.03 \mathrm{eV}$ ) at $49 \mathrm{~ns}$. Such a red shifted emission band at $77 \mathrm{~K}$ can be explained by time resolved phosphorescence measurements, which is discussed in a later section of this paper. It is observed that the emission spectra recorded from $87 \mathrm{~ns}$ to $127 \mathrm{~ms}$ at $77 \mathrm{~K}$ is largely consistent with the previous spectra obtained at $45 \mathrm{~ns}$, indicating that no new excited states are evolved.

In order to understand the complex nature of both triplet emissions (BRTP/BTADF), we performed TRES at RT $\left(\lambda_{\text {ex }}=\right.$ $370 \mathrm{~nm}$ ) (Fig. 6). Both molecules (6.8 $\mu$ s detector delay) show an emission band (FWHM $=\sim 72 \mathrm{~nm})$ at $\sim 457 \mathrm{~nm}(3.0 \mathrm{eV})$ at an early decay time of $7.7 \mu \mathrm{s}$ which matches well with the steady state emission observed at RT (Fig. S10, ESI $\dagger$ ). On a later time scale $(180 \mu \mathrm{s})$, the emission bands progressively blue shift to $\sim 3.06 \mathrm{eV}$ and $3.03 \mathrm{eV}$ with a reduction of FWHM $(\sim 60 \mathrm{~nm}$, $68 \mathrm{~nm}$ ) in both cases (Fig. S12, ESI†). Moreover, the blue shifted emission spectra obtained on the later time scale resemble the 
Table 1 Photophysical parameters of CPPN and CPPNF

\begin{tabular}{|c|c|c|c|c|c|c|c|c|c|c|c|c|}
\hline & \multicolumn{6}{|l|}{ PMMA } & \multicolumn{6}{|l|}{ DPEPO } \\
\hline & $\lambda_{\mathrm{F}}(\mathrm{nm})$ & $\lambda_{\mathrm{P}}(\mathrm{nm})$ & ${ }^{a} \tau_{\mathrm{F}}(\mathrm{ns}, \mu \mathrm{s})$ & ${ }^{a} \tau_{\mathrm{P}}(\mu \mathrm{s})$ & $\Phi_{\mathrm{F}}(\%)$ & ${ }^{b} \Phi_{\mathrm{P}}(\%)$ & ${ }^{a} \lambda_{\mathrm{F}}(\mathrm{nm})$ & $\lambda_{\mathrm{P}}(\mathrm{nm})$ & ${ }^{a} \tau_{\mathrm{F}}(\mathrm{ns}, \mu \mathrm{s})$ & ${ }^{a} \tau_{\mathrm{P}}(\mathrm{ms})$ & $\Phi_{\mathrm{F}}(\%)$ & ${ }^{b} \Phi_{\mathrm{P}}(\%)$ \\
\hline CPPN & 463 & 455 & $13.01,12.8^{c}$ & $58.4,175.0,619.3$ & 43.7 & 3.7 & 475 & 465 & $18.7,5.9^{c}$ & $0.49,5.50,39.30$ & 10.9 & 10.1 \\
\hline CPPNF & 463 & 452 & $15.5,10.4^{c}$ & $148.6,570.0,44.5$ & 42.3 & 3.0 & 484 & 463 & $19.8,4.9^{c}$ & $40.4,3.10$ & 13.2 & 9.8 \\
\hline
\end{tabular}

${ }^{a} \lambda_{\mathrm{ex}}=350 \mathrm{~nm}$ (steady-state and phosphorescence spectra), $370 \mathrm{~nm}$ (lifetime). ${ }^{b}$ Phosphorescence quantum yield under ambient conditions $\left(\lambda_{\mathrm{ex}}=\right.$ $350 \mathrm{~nm}) .{ }^{c}$ Lifetimes $(\mu \mathrm{s})$.

BRTP peak obtained after $50 \mu$ s of the detector delay (Fig. S13, ESI $\dagger$ ). This efficacy confirms that the excited species originated initially after $6.8 \mu \mathrm{s}$ of detector delay is singlet in nature that contributes to the TADF, while the blue-shifted species observed on the later time scale originates from the higher-energy triplet state (BRTP). The time resolved area normalization spectra (TRANES) recorded in the time range of 7.7-180 $\mu$ s also show an isoemissive point thus confirming the involvement of two emissive species due to DF and RTP (Fig. S14, ESI $\dagger$ ). When we compare the TRES data at $7.7 \mu$ s and $49 \mathrm{~ns}$ (RT), we observed that both spectra are similar to each other, which indicates that the emission occurs from the same ${ }^{1} \mathrm{CT}$ state that leads to delayed emission (Fig. S12, ESI $\dagger$ ). At $77 \mathrm{~K}$, the TRES measurements (3.4 $\mu \mathrm{s})$ show no shift in the broad emission band $(\sim 473 \mathrm{~nm})$, while even after a further delay of $110 \mathrm{~ms}$ the phosphorescence emission band remained largely consistent with the emission recorded at a lower delay $(3.4 \mu \mathrm{s})$, ensuring that only one ${ }^{3} \mathrm{CT}$ state was involved in the emission (Fig. S15, ESI $\dagger$ ). Based on the observations of narrow-RTP bands, delayed fluorescence (7.7 $\mu \mathrm{s}, \mathrm{RT})$ and phosphorescence recorded at $77 \mathrm{~K}$, we confirmed that three radiative excited states (two triplets, one singlet) are involved in the emission. To understand the origin of the BRTP bands, we synthesized two control compounds (Fig. 2) in which only phenoxy (PPN) or 2,6-difluorophenoxy (PPNF) groups are covalently attached to the 4,5-positions of the PN core. $0.1 \%$ PMMA films of these compounds ( $\lambda_{\mathrm{ex}}=330 \mathrm{~nm}, 50 \mu \mathrm{s}$ delay) show an emission at $\sim 455 \mathrm{~nm}\left(\tau_{\mathrm{PPN}}=56.20 \mu \mathrm{s}\right.$ and $343.47 \mu \mathrm{s}$ and $\tau_{\mathrm{PPNF}}=197.77 \mu \mathrm{s}$ and $1.58 \mathrm{~ms}$ ) which closely overlaps with the BRTP peak of the parent compounds recorded at RT (Fig. 7 and Fig. S16, ESI $\dagger$ ). At $77 \mathrm{~K}$, in contrast to the parent compounds whose phosphorescence band was missing at $\sim 455 \mathrm{~nm}\left(\lambda_{\mathrm{ex}}=\right.$ $350 \mathrm{~nm})$, a clear vibrational feature $(435,455,478 \mathrm{~nm})$ was observed when they were excited at $290 \mathrm{~nm}$, thus confirming the ${ }^{3} \mathrm{LE}$ nature of the higher energy BRTP bands (PPN, $3.01 \mathrm{eV}$;
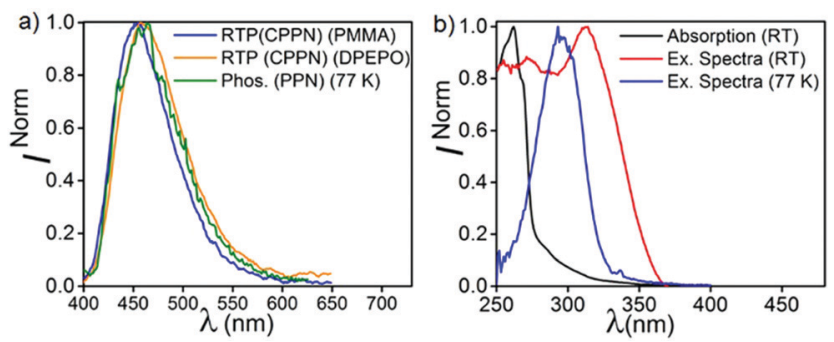

Fig. 7 (a) RTP of CPPN in PMMA and DPEPO under ambient conditions, and phosphorescence of PPN at $77 \mathrm{~K}$. (b) Absorption and excitation spectra of PPN at RT, and excitation spectra of PPN at $77 \mathrm{~K}$.
PPNF, $3.03 \mathrm{eV}$ ) (Fig. S16, ESI $\dagger$ ). When we recorded the absorption and excitation spectra of both PPN and PPNF, we found that (i) the excitation spectra $\left(\lambda_{\mathrm{em}}=390-410 \mathrm{~nm}\right)$ recorded at RT do not overlap with the lower energy region of the absorption spectra, (ii) both absorption band (RT) and excitation spectra (77 K) do not overlap with each other, and (iii) the intensity of the excitation band at $350 \mathrm{~nm}$ is negligible (77 K) (Fig. 7 and Fig. S16, ESI $\dagger$ ). We speculate that the excited states caused by PPN parts of the molecules are not populated when they are excited at $350 \mathrm{~nm}$, while exciting at $290 \mathrm{~nm}$ a new weak phosphorescence emission band at $390-410 \mathrm{~nm}$ (77 K) was observed due to the spectral differences observed in the absorption and excitation spectra discussed above (Fig. S18, ESI $\dagger$ ). Comparing the phosphorescence characteristics of both parent compounds measured at RT with the control compounds, we confirm that the higher energy BRTP peak originates from the PPN part $\left(\mathrm{T}_{\mathrm{PPN}}, \sim 452-455 \mathrm{~nm}\right)$ of both parent molecular systems, while the CzPN part is responsible for the lower energy triplet state $\left(\mathrm{T}_{\mathrm{CzPN}}, \sim 473 \mathrm{~nm}\right)$ that up-converts into the singlet state via the TADF process. ${ }^{15}$

The PL measurements of the parent compounds (PMMA) are summarized in Table 1 and Table S3 (ESI $\dagger$ ). The energy gaps $\left(\Delta E_{\mathrm{S}-\mathrm{T}}{ }^{1}:{ }^{1} \mathrm{CT}_{\mathrm{CzPN}}-{ }^{3} \mathrm{LE}_{\mathrm{PPN}}, \Delta E_{\mathrm{S}-\mathrm{T}}{ }^{2}:{ }^{1} \mathrm{CT}_{\mathrm{CzPN}}-{ }^{3} \mathrm{CT}_{\mathrm{CzPN}}, \Delta E_{\mathrm{T}-\mathrm{T}}\right.$ : $\left.{ }^{3} \mathrm{LE}_{\mathrm{PPN}}-{ }^{3} \mathrm{CT}_{\mathrm{CzPN}}\right)$ between the singlet and triple states of both compounds were calculated to be 0.04, 0.06 and $0.10 \mathrm{eV}$ (CPPN), and $0.05,0.02$ and $0.07 \mathrm{eV}$ (CPPNF). We speculate that such close proximity of the energy levels $\left({ }^{3} \mathrm{LE},{ }^{1} \mathrm{CT},{ }^{3} \mathrm{CT}\right.$ ) (Fig. 8) plays an important role to observe TADF via intermediate statemixing caused by vibronic-coupling between the upper ${ }^{3} \mathrm{LE}$ and lower-lying ${ }^{3} \mathrm{CT}$ states. ${ }^{13,14}$

\subsection{Emission characteristics in DPEPO films}

To gain more insight into the emission behavior, PL studies of bis[2-(di-(phenyl)phosphino)-phenyl]ether oxide (DPEPO) films $(0.1 \%)$ were undertaken. The steady state PL characteristics of both compounds in DPEPO films under ambient conditions are very similar as compared to PMMA films. However, a bathochromic shift (CPPN, $\lambda_{\mathrm{em}}^{\mathrm{ss}}=475 \mathrm{~nm}, \lambda_{\mathrm{em}}^{\mathrm{Phos}}=465 \mathrm{~nm}$; CPPNF, $\left.\lambda_{\mathrm{em}}^{\mathrm{ss}}=484 \mathrm{~nm}, \lambda_{\mathrm{em}}^{\mathrm{Phos}}=463 \mathrm{~nm}\right)$ is observed as compared to PMMA due to the increased polarity of the host material (Fig. S19, ESI $\dagger$ ). The PLQY values were found to be $21 \%$ (CPPN) and $23.2 \%$ (CPPNF) while the phosphorescence quantum yield $\left(\Phi_{\mathrm{P}}\right)$ was increased to $10.04 \%$ and $9.96 \%$, respectively. Fluorescence lifetime analysis of the band (CPPN: $\lambda_{475}, \tau_{\mathrm{PF}}=18.78 \mathrm{~ns}$, $\tau_{\mathrm{DF}}=5.9 \mu \mathrm{s}$; CPPNF: $\left.\lambda_{475}, \tau_{\mathrm{PF}}=19.8 \mathrm{~ns}, \tau_{\mathrm{DF}}=4.99 \mu \mathrm{s}\right)$ under ambient conditions further confirms the TADF feature of the dyes (Fig. S20, ESI $\dagger$ and Table 1). The phosphorescence 
a)

${ }^{1} \mathrm{LE}(3.11 \mathrm{eV})$

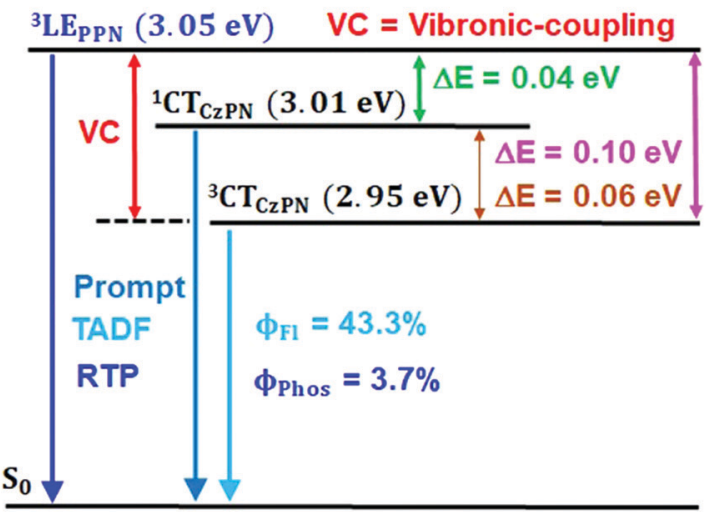

b)

${ }^{1} \mathrm{LE}(3.11 \mathrm{eV})$

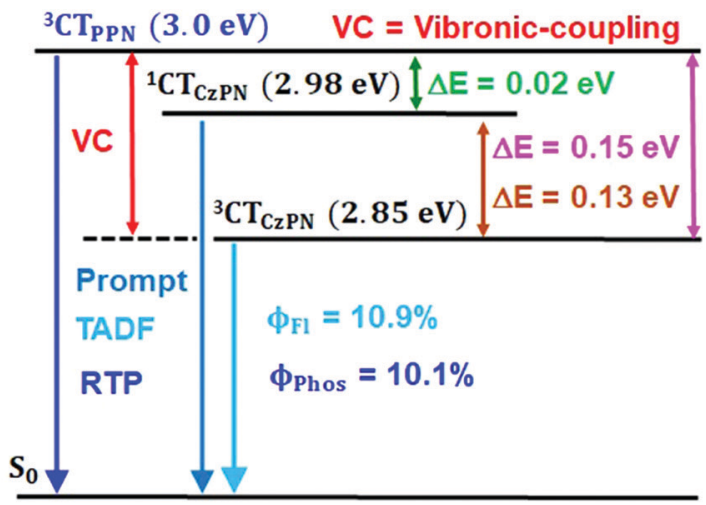

Fig. 8 Jablonski diagrams of CPPN in (a) PMMA (0.1\%) and (b) DPEPO (0.1\%) films.

lifetimes of $\lambda_{462}$ are found to be $59.61 \mathrm{~ms}$ and $5.16 \mathrm{~ms}$ (CPPN), and $68.17 \mathrm{~ms}$ and $5.4 \mathrm{~ms}$ (CPPNF) which are significantly long as compared to the values obtained in PMMA films under ambient conditions (Fig. S21, ESI $\dagger$ ). Such long lifetimes of up to milliseconds further prove the origin of the RTP in these molecules. ${ }^{50}$ Interestingly, RTP lifetimes are substantially reduced with the increasing concentration of the dopants. Such increase in the $\Phi_{\mathrm{P}}$ values and decrease in the RTP lifetimes may originate due to the strong intermolecular interactions between the dopant and the host material which contribute to the reduction of the nonradiative decay through vibrations. ${ }^{41}$ Furthermore, the TRES measurements of $0.1 \%$ DPEPO films under ambient conditions are found to be similar to the results obtained in the PMMA films (Fig. S23-S25, ESI $\dagger$ ). Interestingly, we observed that the emission band is red-shifted $(460 \mathrm{~nm})$ with a broadband signature (FWHM $=76-80 \mathrm{~nm}$ ) as compared to the emission measured in PMMA (Fig. S19, ESI $\dagger$ ), which suggests that the nature of the higher-lying triplet state $\left({ }^{3} \mathrm{LE}_{\mathrm{PPN}}\right)$ in PMMA is changed to ${ }^{3} \mathrm{CT}_{\mathrm{PPN}}(3.0 \mathrm{eV}$, both compounds) in DPEPO under ambient conditions. The phenoxy-phthalonitrile part of CPPN and CPPNF acts as the weak donor-acceptor part as compared to the carbazole-phthalonitrile part due to the presence of weak phenoxy donors. Therefore, a change in the environment from PMMA to DPEPO switches the nature of the triplet state from ${ }^{3} \mathrm{LE}$ to ${ }^{3} \mathrm{CT}$. At $77 \mathrm{~K}$, we observed a single red shifted phosphorescence band $(50 \mu$ s delay) at $490 \mathrm{~nm}$ $(2.85 \mathrm{eV})$ which is similar to the PMMA films (Fig. S25, ESI $\dagger$ ). The DPEPO film studies further ensure that three excited states $\left({ }^{3} \mathrm{CT}_{\mathrm{PPN}},{ }^{1} \mathrm{CT}_{\mathrm{CZPN}},{ }^{3} \mathrm{CT}_{\mathrm{CZPN}}\right)$ are involved in the emission, which is in line with the results obtained in PMMA except for the change in the nature of the higher-lying triplet state (Fig. 8). The energy of the singlet states $\left({ }^{1} \mathrm{CT}_{\mathrm{CzPN}}\right)$ was calculated to be $2.98 \mathrm{eV}$ (CPPN) and $2.94 \mathrm{eV}$ (CPPNF) whereas the triplet state energy $\left({ }^{3} \mathrm{CT}_{\mathrm{CzPN}}, \mathrm{TADF}\right)$ is found to be $2.85 \mathrm{eV}$ for both molecules (Fig. S25 and Table S3, ESI $\dagger$ ). The $\Delta E_{\mathrm{ST}}$ (TADF) is calculated to be $0.13 \mathrm{eV}$ (CPPN) and $0.09 \mathrm{eV}$ (CPPNF). Based on the observations of alteration of the ${ }^{3} \mathrm{LE}$ state to the ${ }^{3} \mathrm{CT}$ state along with high $\Delta E_{\mathrm{ST}}$ and low PLQY values, we reason that mixing of the excited triplet states (RTP, TADF) through vibronic-coupling is substantially reduced in polar DPEPO films as compared to that observed in PMMA due to which substantial reduction in the fluorescence quantum yield was observed. In spite of this, mixing of the intermediate state $\left({ }^{3} \mathrm{CT}_{\mathrm{PPN}}\right)$ contributes to the rISC process which further leads to the TADF emission in DPEPO films as reported by Noda et al. ${ }^{15}$ Comparing photophysical analyses both in PMMA and DPEPO films, we conclude that the higher-energy triplet state originating from the PPN/PPNF part of the molecules acts as an intermediate state that contributes independently as BRTP and BTADF via spin-vibronic coupling. Our studies further confirm that the nature of the higher-lying triplet state plays a decisive role in observing highly efficient simultaneous BRTP and BTADF in $\mathrm{D}_{2} \mathrm{D}_{2}{ }^{\prime}-\mathrm{A}$ conjugates. Besides, the selection of suitable host materials is a requisite to observe such photophysical properties of the molecule.

\subsection{Emission characteristics in PVA films and crystals}

In order to understand the effect of hydrogen bonding ( $\mathrm{H}-\mathrm{B})$ on the photophysical properties, CPPNF was studied in polyvinyl alcohol (PVA) $(0.1 \%, \mathrm{w} / \mathrm{w})$. The steady state and phosphorescence emission bands were observed at $480 \mathrm{~nm}\left(\tau_{\mathrm{PF}}=9.85 \mathrm{~ns}\right.$ and $\left.\tau_{\mathrm{DF}}=4.25 \mu \mathrm{s}\right)$ and $470 \mathrm{~nm}\left(\tau_{\mathrm{P}}=39.51 \mathrm{~ms}, 269.21 \mathrm{~ms}\right)$, respectively (Fig. S26, ESI $\dagger$ ). TRES measurements of the PVA films without detector delay and with a delay of $3.4 \mu$ s showed similar emission behaviour as compared to both in PMMA and DPEPO films (Fig. 9), which further confirms TADF associated in the PVA matrix. However, when the delay was increased to $1.75 \mathrm{~ms}$ (Fig. S27, ESI $\dagger$ ), a broad emission band was observed at $470 \mathrm{~nm}$ (3.06 eV, $2.2 \mathrm{~ms}$ ) caused by the PPNF part of the molecule. On a later time scale $(10.6 \mathrm{~ms})$, the emission band was further red shifted to $525 \mathrm{~nm}(2.79 \mathrm{eV})$ with a reduction in intensity of the higher-lying emission band (3.06 eV), which is inconsistent with the emission behaviour observed in both PMMA and DPEPO films where no such red shifted emission band was observed. However, comparing this observation with the phosphorescence spectra $(50 \mu$ s detector delay) of both 

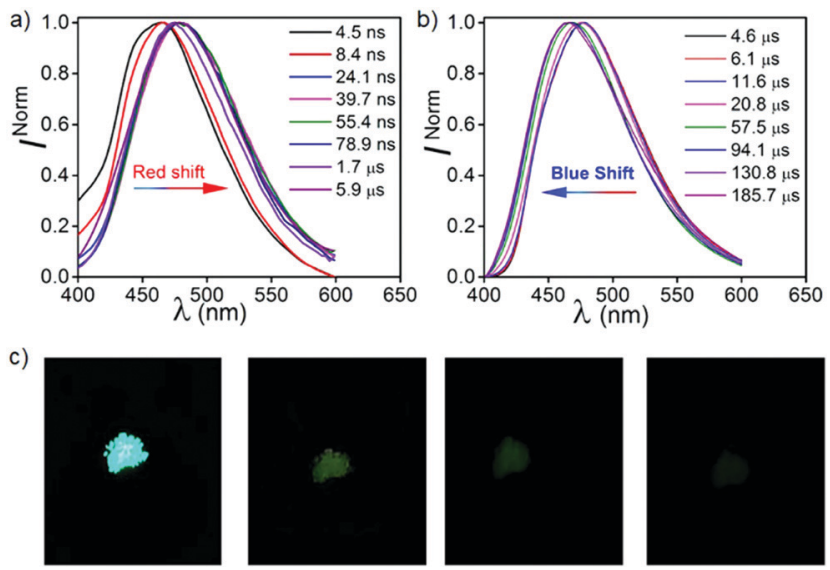

UV on

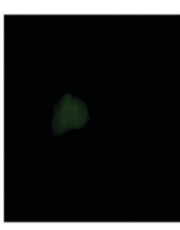

UV off

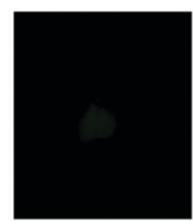

UV off

Fig. 9 TRES of CPPN in PVA $(0.1 \%)$ at (a) without detector delay except the spectrum recorded at time 1.7-5.9 $\mu$ s and (b) $3.4 \mu$ s detector delay under ambient conditions. (c) Images showing PRTP in CPPNF crystals $\left(\lambda_{\mathrm{ex}}=370 \mathrm{~nm}\right)$.

powder $\left(\lambda_{\mathrm{em}}, 510 \mathrm{~nm} ; \tau_{\mathrm{P}}=71.83 \mathrm{~ms}, 187.39 \mathrm{~ms}\right)$ and crystals $\left(\lambda_{\mathrm{em}}, 515 \mathrm{~nm} ; \tau_{\mathrm{P}}=71.98 \mathrm{~ms}, 173.97 \mathrm{~ms}\right)$ of CPPNF recorded under ambient conditions (Fig. 9c and Fig. S26d, ESI $\dagger$ ), we found that the TRES spectra (recorded at $66.9 \mathrm{~ms}$ in PVA films) closely overlaps with the phosphorescence spectra recorded for powder and crystals (Fig. S27d, ESI $\dagger$ ). Now, it is clear that the further bathochromic shift of the lower energy emission band at $2.79 \mathrm{eV}$ is due to aggregation of the molecules, which leads to the formation of an additional lower energy triplet state. With a $1.75 \mathrm{~ms}$ detector delay in films, we observed an isoemissive point in the TRANES spectra, suggesting that two emissive triplet states are involved. The higher energy RTP band originates due to the PPNF part of the molecule while the lower energy emission is due to the new triplet state formed via aggregation (see the $\mathrm{ESI} \dagger$ for details). Therefore, three triplet states $\left(\mathrm{T}_{\mathrm{PPNF}}, \mathrm{T}_{\mathrm{CzPN}}, \mathrm{T}_{\mathrm{agg}}\right)$ are responsible for the emission. Furthermore, the persistent-RTP obtained in films can be explained by the stabilization of the triplet states caused by both aggregation of CPPNF, and H-B interactions between the $\mathrm{F}$ atoms of CPPNF and -OH groups present in PVA. ${ }^{30,33}$

Considering the PL studies of all the films (0.1\%) (PMMA, DPEPO, PVA), we believe that the phenoxy groups covalently connected at the 4,5-positions of the phthalonitrile acceptor cause blue-RTP, while blue-TADF is observed due to the presence of carbazolyl groups attached to the 3,6-positions of the acceptor core. Moreover, the precise substitution of the phenoxy donors can lead to persistent RTP in different host materials and crystals.

\section{Conclusions}

In summary, we have demonstrated simultaneous emission via BRTP and BTADF from carbazolyl-phenoxy-phthalonitrile based donor $\left(\mathrm{D}_{2} \mathrm{D}_{2}{ }^{\prime}\right)$-acceptor (A) conjugates. Experimental results provide unequivocal evidence of BRTP via radiative decay of the

higher-lying triplet state from the phenoxy-phthalonitrile (PPN) part, while TADF occurs from the carbazolyl-phthalonitrile (CzPN) part utilizing the triplet state of PPN via spin-vibronic coupling. Moreover, both blue-RTP and blue-TADF characteristics can be tuned using different host materials and chemical modification of the phenoxy rings. This design strategy may lead to the enhancement of our understanding of highly efficient intrinsic simultaneous emission via RTP and TADF. These systems with asymmetric donors can be further developed to understand the complex exciton dynamics of both singlet and triplet states essential for security applications, imaging and sensors.

\section{Conflicts of interest}

There are no conflicts to declare.

\section{Acknowledgements}

D. R. is grateful to the Board of Research and Nuclear Science (BRNS) (File No. 37(3)/14/08-2018-BRNS/37130), DAE; and Shiv Nadar University (SNU) for generous support. H. B. thanks SNU for fellowship.

\section{Notes and references}

1 I. Bhattacharjee, N. Acharya, S. Karmakar and D. Ray, J. Phys. Chem. C, 2018, 122, 21589-21597.

2 C. A. DeRosa, S. Hiroto and C. L. Fraser, J. Phys. Chem. C, 2019, 123, 20488-20496.

3 G. Zhang, G. M. Palmer, M. W. Dewhirst and C. L. Fraser, Nat. Mater., 2009, 8, 747-751.

4 A. Pigliucci, P. Nikolov, A. Rehaman, L. Gagliardi, C. J. Cramer and E. Vauthey, J. Phys. Chem. A, 2006, 110, 9988-9994.

5 N. J. Turro, V. Ramamurthy and J. C. Scaiano, Principles of Molecular Photochemistry, University Science Books, Sausalito, CA, 2009.

6 I. Bhattacharjee, N. Acharya, H. Bhatia and D. Ray, J. Phys. Chem. Lett., 2018, 9, 2733-2738.

7 Y. Xu, C. Wang, X. Zhou, J. Zhou, X. Guo, X. Liang, D. Hu, F. Li, D. Ma and Y. Ma, J. Phys. Chem. Lett., 2019, 10, 6878-6884.

8 J. S. Ward, N. A. Kukhta, P. L. dos Santos, D. G. Congrave, A. S. Batsanov, A. P. Monkman and M. R. Bryce, Chem. Mater., 2019, 31, 6684-6695.

9 M. Li, Y.-F. Wang, D. Zhang, L. Duan and C.-F. Chen, Angew. Chem., Int. Ed., 2020, 59, 3500-3504.

10 W. Li, B. Li, X. Cai, L. Gan, Z. Xu, W. Li, K. Liu, D. Chen and S.-J. Su, Angew. Chem., Int. Ed., 2019, 58, 11301-11305.

11 P. Stachelek, J. S. Ward, P. L. dos Santos, A. Danos, M. Colella, N. Haase, S. J. Raynes, A. S. Batsanov, M. R. Bryce and A. P. Monkman, ACS Appl. Mater. Interfaces, 2019, 11, 27125-27133.

12 X.-K. Chen, D. Kim and J.-L. Brédas, Acc. Chem. Res., 2018, 51, 2215-2224. 
13 M. K. Etherington, J. Gibson, H. F. Higginbotham, T. J. Penfold and A. P. Monkman, Nat. Commun., 2016, 7, 13680.

14 J. Gibson, A. P. Monkman and T. J. Penfold, ChemPhysChem, 2016, 17, 2956-2961.

15 H. Noda, X.-K. Chen, H. Nakanotani, T. Hosokai, M. Miyajima, N. Notsuka, Y. Kashima, J.-L. Brédas and C. Adachi, Nat. Mater., 2019, 18, 1084-1090.

16 Y. Olivier, M. Moral, L. Muccioli and J.-C. Sancho-García, J. Mater. Chem. C, 2017, 5, 5718-5729.

17 P. K. Samanta, D. Kim, V. Coropceanu and J.-L. Brédas, J. Am. Chem. Soc., 2017, 139, 4042-4051.

18 M. A. Baldo, D. F. O'Brien, Y. You, A. Shoustikov, S. Sibley, M. E. Thompson and S. R. Forrest, Nature, 1998, 395, 151-154.

19 C. Fan and C. Yang, Chem. Soc. Rev., 2014, 43, 6439-6469.

20 A. Fermi, G. Bergamini, M. Roy, M. Gingras and P. Ceroni, J. Am. Chem. Soc., 2014, 136, 6395-6400.

21 M. Shimizu, R. Shigitani, M. Nakatani, K. Kuwabara, Y. Miyake, K. Tajima, H. Sakai and T. Hasobe, J. Phys. Chem. C, 2016, 120, 11631-11639.

22 H. Zhao, L. Zang, H. Zhao, F. Qin, Z. Li, Z. Zhang and W. Cao, J. Phys. Chem. C, 2015, 119, 10558-10563.

23 G. Chen, H. Feng, F. Feng, P. Xu, J. Xu, S. Pan and Z. Qian, J. Phys. Chem. Lett., 2018, 9, 6305-6311.

24 L. Gu, H. Shi, L. Bian, M. Gu, K. Ling, X. Wang, H. Ma, S. Cai, W. Ning, L. Fu, H. Wang, S. Wang, Y. Gao, W. Yao, F. Huo, Y. Tao, Z. An, X. Liu and W. Huang, Nat. Photonics, 2019, 13, 406-411.

25 S. Hirata, J. Mater. Chem. C, 2018, 6, 11785-11794.

26 S. Hirata and M. Vacha, Adv. Opt. Mater., 2017, 5, 1600996.

27 M. S. Kwon, D. Lee, S. Seo, J. Jung and J. Kim, Angew. Chem. Int. Ed., 2014, 53, 11177-11181.

28 X. Ma, J. Wang and H. Tian, Acc. Chem. Res., 2019, 52, 738-748.

29 Y. Xu, W. Yang, D. Yao, K. Bian, W. Zeng, K. Liu, D. Wang and B. Zhang, Chem. Sci., 2020, 11, 419-428.

30 H. Bhatia, I. Bhattacharjee and D. Ray, J. Phys. Chem. Lett., 2018, 9, 3808-3813.

31 H. Bhatia and D. Ray, J. Phys. Chem. C, 2019, 123, 22104-22113.

32 G. Baryshnikov, B. Minaev and H. Ågren, Chem. Rev., 2017, 117, 6500-6537.

33 A. Köhler and H. Bässler, Mater. Sci. Eng., R, 2009, 66, 71-109.
34 S. K. Lower and M. A. El-Sayed, Chem. Rev., 1966, 66, 199-241.

35 T. J. Penfold, E. Gindensperger, C. Daniel and C. M. Marian, Chem. Rev., 2018, 118, 6975-7025.

36 S. Hirata, J. Phys. Chem. Lett., 2018, 9, 4251-4259.

37 C. S. Bilen, N. Harrison and D. J. Morantz, Nature, 1978, 271, 235-237.

38 E. Lucenti, A. Forni, C. Botta, L. Carlucci, C. Giannini, D. Marinotto, A. Pavanello, A. Previtali, S. Righetto and E. Cariati, Angew. Chem., Int. Ed., 2017, 56, 16302-16307.

39 Z. An, C. Zheng, Y. Tao, R. Chen, H. Shi, T. Chen, Z. Wang, H. Li, R. Deng, X. Liu and W. Huang, Nat. Mater., 2015, 14, 685-690.

40 J. Zhang, E. Sharman, L. Yang, J. Jiang and G. Zhang, J. Phys. Chem. C, 2018, 122, 25796-25803.

41 Y. Lei, W. Dai, Y. Tian, J. Yang, P. Li, J. Shi, B. Tong, Z. Cai and Y. Dong, J. Phys. Chem. Lett., 2019, 10, 6019-6025.

42 R. Kabe, N. Notsuka, K. Yoshida and C. Adachi, Adv. Mater., 2016, 28, 655-660.

43 R. Huang, J. Avó, T. Northey, E. Chaning-Pearce, P. L. dos Santos, J. S. Ward, P. Data, M. K. Etherington, M. A. Fox, T. J. Penfold, M. N. Berberan-Santos, J. C. Lima, M. R. Bryce and F. B. Dias, J. Mater. Chem. C, 2017, 5, 6269-6280.

44 J. S. Ward, R. S. Nobuyasu, A. S. Batsanov, P. Data, A. P. Monkman, F. B. Dias and M. R. Bryce, Chem. Commun., 2016, 52, 2612-2615.

45 I. Bhattacharjee, N. Acharya and D. Ray, Chem. Commun., 2019, 55, 1899-1902.

46 L. Zhan, Z. Chen, S. Gong, Y. Xiang, F. Ni, X. Zeng, G. Xie and C. Yang, Angew. Chem. Int. Ed., 2019, 58, 17651-17655.

47 P. Data, M. Okazaki, S. Minakata and Y. Takeda, J. Mater. Chem. C, 2019, 7, 6616-6621.

48 T. Serevičius, T. Bučiūnas, J. Bucevičius, J. Dodonova, S. Tumkevičius, K. Kazlauskas and S. Juršènas, J. Mater. Chem. C, 2018, 6, 11128-11136.

49 Y. Takeda, T. Kaihara, M. Okazaki, H. Higginbotham, P. Data, N. Tohnai and S. Minakata, Chem. Commun., 2018, 54, 6847-6850.

50 A. Tomkeviciene, T. Matulaitis, M. Guzauskas, V. Andruleviciene, D. Volyniuk and J. V. Grazulevicius, Org. Electron., 2019, 70, 227-239. 